\title{
Image Watermarking Based on Fourier-Mellin Transform
}

\author{
Khadija Gourrame ${ }^{1,2(凶)}$, Hassan Douzi ${ }^{1}$, Rachid Harba ${ }^{2}$, \\ Riad Rabia ${ }^{1}$, Frederic Ros ${ }^{2}$, and Mehamed ElHajji ${ }^{1}$ \\ ${ }^{1}$ IRF-SIC Laboratory, Ibn Zohr University, Agadir, Morocco \\ khadija.gourrame@edu.uiz.ac.ma \\ 2 PRISME Laboratory, Orleans University, Orleans, France
}

\begin{abstract}
Geometric attacks are still challenging issues in image watermarking. In this paper, the robustness of different insertion position and shape of the watermark are evaluated in watermarking scheme based on Fourier-Mellin transform. We propose diagonal, rectangular, and circular insertion of the mark. The robustness of these techniques against geometric deformations such as rotation, scaling and translation (RST) is evaluated. Results show that the circular insertion performs better for translation and scaling attacks, while the diagonal insertion is better for rotations and RST attacks. The last point makes the diagonal insertion to be preferred in industrial applications since the combination of RST attacks often occurs in many applications such as printing the image on a physical support, and scanning it (print-scan attack).
\end{abstract}

Keywords: Image watermarking $\cdot$ Synchronization $\cdot$ Fourier-Mellin transform $\cdot$ RST

\section{Introduction}

Robustness of image watermarking techniques is an active research area for many years. Yet, there is no existing watermarking method that achieves a complete robustness to all common watermarking attacks including geometric distortions. In general, image watermarking techniques are implemented in spatial domain or transformed domains [1]. The most used transforms are discrete cosine transform (DCT), discrete wavelet transform (DWT), and discrete Fourier transform (DFT). Although DCT and DWT are very robust to common signal processing attacks such as compression and signal filtering, they are weak to resist geometric distortions [2]. However, DFT has the advantage of being more resistant to geometric attacks [3, 4].

Geometric distortions are one of the major issues in industrial watermarking applications where the so-called print-scan occurs (print-scan means that the image is printed on a physical support, and then scanned). RST (rotation, scaling and translation) deformations are typically produced by print-scan process. Geometric attacks cause a loss of synchronization between the extracted watermark and the embedded one. Thus, many schemes are proposed in the literature to solve the synchronization issue. 
In our team, Riad et al. have developed a robust watermarking method in the Fourier domain for print-scan process using a Fourier method for identity (ID) images [4]. The object was to increase the security of official documents as for example ID cards or passports. In that case, the print-scan attack produces rotations and translations of small amplitudes. To be rotation invariant, the watermark vector is embedded in the magnitude of the Fourier transform of the original image, as a circle of radius $r$ around the center of the transformed image in the medium frequency domain, translation problems are being naturally handled by Fourier capabilities. Regarding scaling deformations, in $[3,4]$, they proposed to resize the image to its original size before the watermark detection. However, this Fourier method is not robust to scale deformations in case if the size of original image is unknown. To overcome the scaling problem, Fourier log-polar approach or Fourier-Mellin transform [5, 6] is proposed as an RST invariant method. Converting the cartesian coordinates of Fourier magnitude into logpolar coordinates, transforms the rotation and scaling operations into translation ones, so that the whole process becomes invariant to RST. Many watermarking techniques are proposed in literature using FMT. Many research works try to reduce the steps in Fourier-Mellin transform as in [6]. Log-polar transform (LPT) is used as a partial step of FMT technique for proposing robust image watermarking [7], however this proposition is designed for non-blind watermarking method. Fourier-Mellin moments are used as image features for watermark registration and extraction for copyright authentication [8]. Quaternion Discrete Fourier transform (QDFT) combined with LPM represents new version of FMT in recent years [9], this method is robust against signal processing attacks for small image blocks with QDFT. However, for geometric attacks, the robustness was secured with the help of a template, which limits and reduces the range of RST attacks. After the O. Ruanaidh and T. Pun [5] work, the followed proposed method in literature try to avoid to go through all the steps to get an RST invariant domain, which it compromise on the robustness of the method in the first place. Early studies show that considering shape, position and other parameters related to the watermark are improving on the robustness of the method $[4,10]$.

In this paper, different insertion watermarking schemes based on Fourier-Mellin transform (FMT) are studied. These techniques are tested against geometric deformation such as translation, rotation and scaling (RST). The methods associate watermark insertion in different geometrical shapes and positions in invariant RST domains based on Fourier-Mellin transform. The presented techniques are circular insertion [4], diagonal insertion [10] and rectangular insertion. The robustness of the presented methods are compared and discussed.

The paper is organized as follows. Section 2 presents the theoretical background. Section 4 describes the watermarking method based on FMT and it shows results of the robustness test of the three tested methods under RTS attacks, Sect. 4 gives a conclusion. 


\section{Fourier-Mellin Transform - Mathematical Background}

In this section, geometric properties of the Fourier transform are presented. The properties of the Fourier-Mellin transform are also explained which show that it is naturally resistant to RST.

\subsection{Fourier Transform}

The Fourier transform $F(u, v)$ of an image $f(x, y)$ is:

$$
\mathrm{f}(\mathrm{x}, \mathrm{y}) \stackrel{\mathrm{FT}}{\leftrightarrow}\left(\mathrm{F}(\mathrm{u}, \mathrm{v})=\int_{-\infty}^{\infty} \int_{-\infty}^{\infty} \mathrm{f}(\mathrm{x}, \mathrm{y}) \mathrm{e}^{-\mathrm{j} 2 \pi(\mathrm{ux}+\mathrm{vy})} \mathrm{dxdy}\right) .
$$

Ft is invariant to translations in term of magnitude part:

$$
\mathrm{f}(\mathrm{x}-\mathrm{a}, \mathrm{y}-\mathrm{b}) \stackrel{\mathrm{FT}}{\leftrightarrow} \mathrm{e}^{-\mathrm{j} 2 \pi(\mathrm{au}+\mathrm{bv})} \mathrm{F}(\mathrm{u}, \mathrm{v}),
$$

where $a$ and $b$ are translation parameters. Rotation of an image with angle $\theta$ in spatial domain is converted into a rotation with the same angle in Fourier domain:

$$
\begin{gathered}
\mathrm{f}(\mathrm{x} \cos (\theta)-\mathrm{y} \sin (\theta), \mathrm{x} \sin (\theta)+\mathrm{y} \cos (\theta)) \\
\mathrm{F}(\mathrm{u} \cos (\theta)-\mathrm{v} \sin (\theta), \mathrm{u} \sin (\theta)+\mathrm{v} \cos (\theta)) .
\end{gathered}
$$

Scaling in spatial domain with a factor $a$ is converted into a scaling with the inverse factor in Fourier domain:

$$
\mathrm{f}(\mathrm{ax}, \mathrm{by}) \stackrel{\mathrm{FT}}{\leftrightarrow} \frac{1}{|\mathrm{ab}|} \mathrm{F}\left(\frac{\mathrm{u}}{\mathrm{a}}, \frac{\mathrm{v}}{\mathrm{b}}\right) .
$$

Properties in (2) and (3) demonstrate that the magnitude of the Fourier transform naturally resists to rotations and translations, but not to scaling changes. It is therefore naturally adapted to RT attacks.

\subsection{Fourier-Mellin Transform}

Fourier-Mellin transform of image $f(\mathrm{r}, \theta)$ (in polar coordinates) is:

$$
\operatorname{FM}(\mathrm{u}, \mathrm{v})=\frac{1}{2 \pi} \int_{0}^{\infty} \int_{0}^{2 \pi} \mathrm{f}(\mathrm{r}, \theta) \mathrm{r}^{-\mathrm{jv}-1} \mathrm{e}^{-\mathrm{ju} \theta} \mathrm{drd} \theta
$$

With a set of integration by substitution, FMT can be expressed as FT of an image in log polar coordinates [5]:

$$
\operatorname{FM}(u, v)=\frac{1}{2 \pi} \int_{0}^{2 \pi} \int_{-\infty}^{\infty} f^{\prime}(\rho, \theta) e^{-j(v \rho+u \theta)} d \rho d \theta=F\left\{f^{\prime}(\rho, \theta)\right\}
$$


The geometric properties of the Fourier-Mellin transform are related to the geometric properties of both the log-polar transform and the Fourier transform:

Log-polar transform converts rotation and scaling into translation as expressed in the following equations:

$$
\begin{gathered}
\left(x^{\prime}, y^{\prime}\right) \leftrightarrow(r, \theta+\varphi) \leftrightarrow(\rho, \theta+\varphi), \\
(a x, b y) \leftrightarrow(a r, \theta) \leftrightarrow(\log (a r), \theta)=(\rho+\log (a), \theta),
\end{gathered}
$$

where $\left(x^{\prime}, y^{\prime}\right)$ are cartesian coordinates after rotation with $\varphi .(r, \theta)$ and $(\rho, \theta)$ are the polar and log-polar coordinates respectively.

Fourier transform is invariant to translation therefore Fourier transform of an image in log-polar coordinates is invariant to rotation and scaling. As a result, Fourier-Mellin transform is invariant to rotation and scaling.

\section{Fourier-Mellin Method's Comparison}

Fourier-Mellin domain for watermarking is described first by O. Ruanaidh and T. Pun [5]. Figure 1. illustrates the process of obtaining the rotation, scaling and translation (RST) transformation invariant from a digital image. This is explained in the following. Imagine that the input image at the bottom of Fig. 1 is subject to a RST attack. As seen in Fig. 1, a series of 3 transforms is going to be applied to this image. For the first FT, the magnitude of the FT of the image is invariant to translation, while a rotation and a scale changes are still present. When the LPT is applied (the first part of the FM transform), the remaining rotation and scale are transformed into translations. All these properties were recalled in Sect. 2 of this document. For the last FT (the second part of the FMT), the magnitude of the FT is now invariant to translations (known from the first FT), but it is also invariant to rotation and scale. It results in a RST invariant domain.

The watermark takes the form of two dimensional spread spectrum signal in the RST transformation invariant domain. First, Fourier transform (FFT) is applied and then followed by a Fourier-Mellin transform (FMT- A log-polar mapping (LPM) followed by a Fourier transform). The invariant coefficients selected for their robustness to image processing are marked using a spread spectrum signal. The inverse mapping is computed as an inverse Fourier transform (IFFT) followed by an inverse Fourier-Mellin transform (IFMT- An inverse log-polar mapping (ILPM) followed by an inverse FFT).

The embedding process of the three methods consists on: First, luminance values of the cover work are transformed to the RST domain Fig. 1. Then the watermark $W$ as a pseudo-random sequence of $\mathrm{N}$ binary elements is inserted in the mid frequencies magnitude coefficients along a circle of radius $r$, which can be expressed with this equation:

$$
M_{W}=M_{f}+\alpha \times W
$$

where $M_{W}$ is the mid frequencies in RST domain Fig. 1, $M_{f}$ is the original frequencies before the watermark insertion, and $\alpha$ is the strength parameter. 


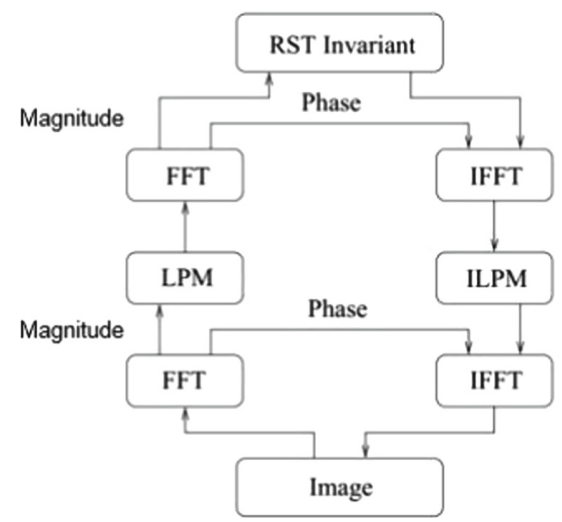

Fig. 1. RST invariant watermarking scheme based on Fourier-Mellin transform [5].

The choice of $\alpha$ is related to the invisibility of the watermark. The watermark is spread over all the image pixels taking into account the peak signal-to-noise ratio (PSNR) metric. Hence, an adaptive strength $\alpha$ is determined to obtain the desired value of PSNR, in general equal to $40 \mathrm{~dB}[3,11]$. Finally, the watermarked image is obtained by applying the inverse process of FMT Fig. 1. The blind detection needs only the tested image and the watermark $W$. First, the FMT is applied to the luminance of the image. Then, the coefficients are extracted from the RST domain. the normalized correlation is computed between the extracted coefficients and the sequence $\mathrm{W}$ of the watermark [2].

\subsection{Circular Insertion of the Watermark}

See Fig. 2 and Table 1.

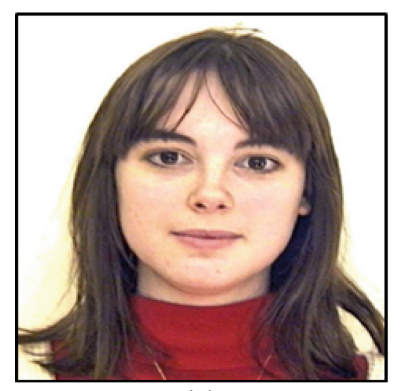

(a)

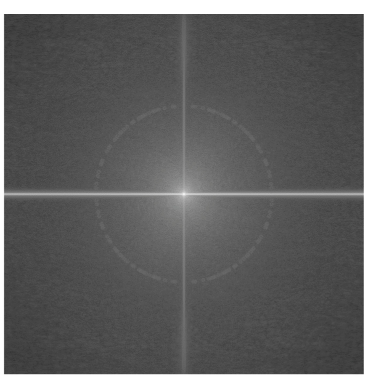

(b)

Fig. 2. (a) Watermarked image and (b) circular watermark insertion in the magnitude of FMT. 
Table 1. PSNR and correlation values of circular insertion

\begin{tabular}{l|l}
\hline PSNR & $40.4007 \mathrm{~dB}$ \\
\hline Correlation value & 0.9750 \\
\hline Correlation without watermark & 0.0822 \\
\hline
\end{tabular}

\subsection{Diagonal Insertion}

See Fig. 3 and Table 2.

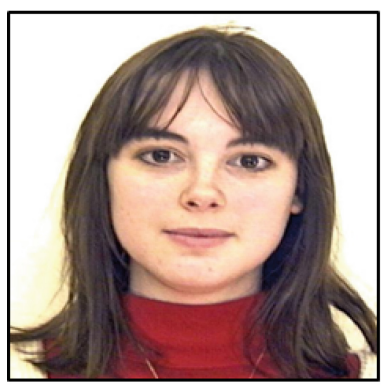

(a)

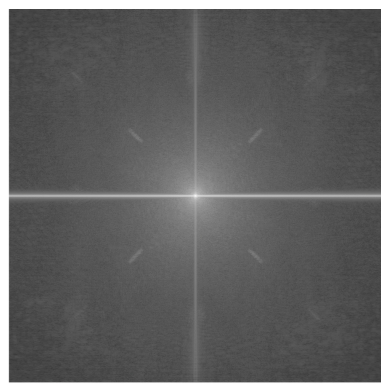

(b)

Fig. 3. (a) Watermarked image and (b) diagonal watermark insertion in the magnitude of FMT.

Table 2. PSNR and correlation values of diagonal insertion

\begin{tabular}{l|l}
\hline PSNR & $40.3173 \mathrm{~dB}$ \\
\hline Correlation value & 0.9182 \\
\hline Correlation without watermark & 0.0346 \\
\hline
\end{tabular}

\subsection{Rectangular Insertion}

See Fig. 4 and Table 3.

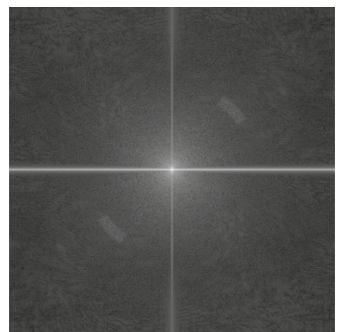

Fig. 4. Rectangular watermark insertion in the magnitude of FMT. 
Table 3. PSNR and correlation values of rectangular insertion

\begin{tabular}{l|l}
\hline PSNR & $40.267 \mathrm{~dB}$ \\
\hline Correlation value & 0.8923 \\
\hline Correlation without watermark & 0.2989 \\
\hline
\end{tabular}

\subsection{Comparison Results}

Comparison results are presented in Fig. 5. Three methods of invisible watermarking based on Fourier-Mellin are tested. As explained earlier, those methods are circular, diagonal and rectangular insertions. Watermarked and unwatermarked image of each method are tested under four types of geometric distortions: Diagonal translations with pixels in the range $[0,100]$, rotations between 1 and $19^{\circ}$, scaling with factors in the range $[0.88,1.24]$ and combination of the above geometric distortions RST.

Under translation attack, the three tested methods give better result with the higher performance of the circular insertion method. Diagonal insertion shows better

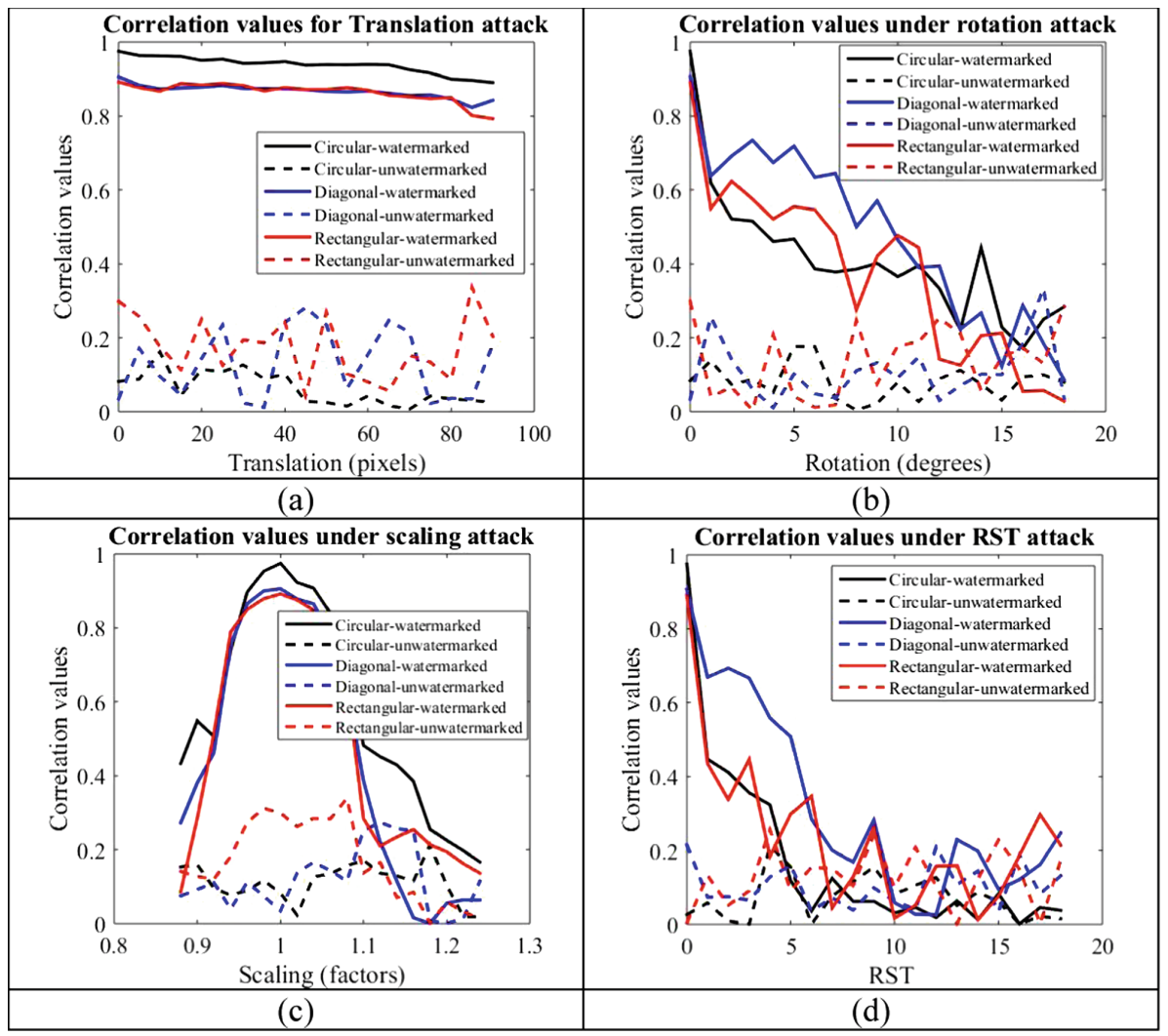

Fig. 5. Correlation values of the three tested methods for watermarked and unwatermarked images with (a) translation attack, (b) rotation attack and (c) scaling attack and (d) RST attack. 
performance against rotation attack compared with circular and diagonal insertion method. However, circular insertion shows higher performance against scaling attack. Under a combination of the attacks RST the performance of the three methods is similar with a slight outperform of diagonal insertion.

\section{Conclusion}

In this paper, the robustness of different insertion position and shape of the watermark were evaluated in a watermarking scheme based on the Fourier-Mellin transform (FMT). The diagonal, rectangular and circular insertions were tested against geometric deformation such as rotation, scaling and translation (RST). Results show that the circular insertion performs better for translations and scaling attacks, while the diagonal insertion is better for rotations and RST attacks. This last point makes that the diagonal insertion should be preferred in industrial applications when printing the image on a physical support, and scanning it (print-scan process or print-scan attack). Future works concerned the protection of official documents containing an Identity image (passport or ID cards) using watermarking methods. In that case, it is expected in the future that the detection of the mark in the ID document will be assessed using a smartphone (print-cam process). The geometric attack is stronger than the RST attack. It is a perspective attack, a RST attack combined to a tilling of the optical axis of the camera that takes the image. In a first step, we will test the robustness of the FMT watermark insertion with various insertion position and shape to the perspective attack. In a second step, we will develop an android version of the method.

\section{References}

1. Barni, M., Cox, I., Kalker, T., Kim, H.-J. (eds.): Digital Watermarking, IWDW 2005. LNCS, vol. 3710. Springer, Heidelberg (2005). https://doi.org/10.1007/11551492

2. Cox, I., Miller, M., Bloom, J., Fridrich, J., Kalker, T.: Digital Watermarking and Steganography. Morgan Kaufmann, Boston (2007)

3. Poljicak, A., Mandic, L., Agic, D.: Discrete Fourier transform-based watermarking method with an optimal implementation radius. J. Electron. Imaging 20(3), 033008 (2011)

4. Riad, R., Harba, R., Douzi, H., Ros, F., Elhajji, M.: Robust Fourier watermarking for id images on smart card plastic supports. Adv. Electr. Comput. Eng. 16(4), 23-30 (2016)

5. Ruanaidh, J.J.O., Pun, T.: Rotation, scale and translation invariant spread spectrum digital image watermarking. Sig. Process. 66(3), 303-317 (1998)

6. Lin, C.-Y., Wu, M., Bloom, J.A., Cox, I.J., Miller, M.L., Lui, Y.M.: Rotation, scale, and translation resilient watermarking for images. IEEE Trans. Image Process. 10(5), 767-782 (2001)

7. Zheng, D., Zhao, J., El Saddik, A.: RST-invariant digital image watermarking based on logpolar mapping and phase correlation. IEEE Trans. Circuits Syst. Video Technol. 13(8), 753765 (2003)

8. Shao, Z., Shang, Y., Zhang, Y., Liu, X., Guo, G.: Robust watermarking using orthogonal Fourier-Mellin moments and chaotic map for double images. Sig. Process. 120, 522-531 (2016) 
9. Ouyang, J., Coatrieux, G., Chen, B., Shu, H.: Color image watermarking based on quaternion Fourier transform and improved uniform log-polar mapping. Comput. Electr. Eng. 46, 419-432 (2015)

10. Kim, B.-S., et al.: Robust digital image watermarking method against geometrical attacks. Real-Time Imaging 9(2), 139-149 (2003)

11. Cheddad, A., Condell, J., Curran, K., Mc Kevitt, P.: Digital image steganography: survey and analysis of current methods. Sig. Process. 90(3), 727-752 (2010) 